\title{
Dietary Fat Intake and Its Relationship with Serum Lipid Profiles in Tehranian Adolescents
}

\author{
Sahar Mohseni-Takalloo ${ }^{1,2}$, Parvin Mirmiran ${ }^{3, *}$, Firoozeh Hosseini-Esfahani ${ }^{1}$, Fereidoun Azizi ${ }^{4}$ \\ ${ }^{1}$ Nutrition and Endocrine Research Center, Research Institute for Endocrine Sciences, Shahid Beheshti University of Medical Sciences, \\ Tehran, Iran \\ ${ }^{2}$ Faculty of School of Medicine, Bam University of Medical Sciences, Bam, Iran \\ ${ }^{3}$ Faculty of Nutrition Sciences and Food Technology, National Nutrition and Food Technology Research Institute, Shahid Beheshti \\ University of Medical Sciences, Tehran, Iran \\ ${ }^{4}$ Endocrine Research Center, Research Institute for Endocrine Sciences, Shahid Beheshti University of Medical Sciences, Tehran, Iran \\ *Corresponding author: Mirmiran@endocrine.ac.ir
}

Received June 03, 2014; Revised June 17, 2014; Accepted June 19, 2014

\begin{abstract}
Dyslipidemia is an important risk factor for cardiovascular disease (CVD) and an important cause of death in adults. Long-term follow-up studies have demonstrated that dietary fat consumption in children and adolescent is one of these risk factors of dyslipidemia in adulthood. The purpose of the current study was to assess the dietary fat intake of Iranian adolescents and their relationship with serum lipid profiles. In this population based cross-sectional study 717 adolescents (391 girls and 326 boys), aged 10 to 19 years, were randomly selected from the fourth phase of Tehran Lipid and Glucose Study (2008-2011). Usual dietary intakes were assessed using valid and reliable food frequency questionnaire and a fasting blood sample was given from all participants to analyze serum lipid profile. Among serum lipid levels, only triglyceride (TG) concentration was higher significantly in boys. In girls, the percent of energy intake from total fat, poly unsaturated fatty acid (PUFA), mono unsaturated fatty acid (MUFA) and trans fatty acids were higher significantly. After adjustment for sex and age, serum levels of TG and HDL-C showed a significant decreasing and increasing trend according to the quartiles of percent of energy intake from PUFA, respectively. No significant difference was observed between other fatty acids and serum lipid profile. In conclusion, although the percent of calorie intake from fat and different types of fatty acids have effect in determining serum lipids levels, but they are not the most important factors for determining the serum lipid profile.
\end{abstract}

Keywords: dietary fat intake, lipid profile, saturated fatty acid, polyunsaturated fatty acid, trans fatty acid, monounsaturated fatty acid

Cite This Article: Sahar Mohseni-Takalloo, Parvin Mirmiran, Firoozeh Hosseini-Esfahani, and Fereidoun Azizi, "Dietary Fat Intake and Its Relationship with Serum Lipid Profiles in Tehranian Adolescents." Journal of Food and Nutrition Research, vol. 2, no. 6 (2014): 330-334. doi: 10.12691/jfnr-2-6-10.

\section{Introduction}

Dyslipidemia is defined as abnormal lipoprotein metabolism, including elevated triglycerides (TG), total cholesterol (TC) and low density lipoprotein cholesterol (LDL-C) or reduced high density lipoprotein cholesterol (HDL-C) [1]. Dyslipidemia is an established risk factor for cardiovascular disease (CVD) -an important cause of death- in adults [1]. Studies of children and young adults indicated early lesions of atherosclerosis (fatty streaks) were occurred in the abdominal aorta at age 3 years, coronary arteries at age 10 years and further progression with at ages 8-12 [2,3]. Although, dyslipidemia is rarely leads to adverse health outcomes in childhood, but its long-term effects are considerable [1]. Long-term followup studies have demonstrated that dietary fat consumption in childhood is one of the risk factors that can have a longterm effect on adult health, particularly the risk of CVD [3].
In adolescents, the initial treatment of dyslipidemia involves the provision of a balanced nutritional intake [4]. The cornerstone of management of lipid abnormalities has focused on the amount and type of fatty acids and cholesterol intake [5]. Previous studies have shown a relationship between intake of saturated fatty acids, polyunsaturated fatty acids, mono unsaturated fatty acids and cholesterol with dyslipidemia and increased risk of CVD [5,6,7,8,9]. Obtained results from previous researches showed that in Iran, the prevalence of CVD risk factors have increased and the age of CVD onset among the population has decreased [10]. Also, they indicated that modifications in childhood lipid profiles could be due to switching rapidly from traditional diet to western diet [9].

The purpose of the current study was to assess the dietary fat intake of Iranian adolescents and their correlation with serum lipid profiles. Such identification will be very useful for designing effective intervention and preventive programs aimed at reducing CVD risk in both population and individualized approaches. 


\section{Methods and Materials}

\subsection{Study Population}

The Tehran Lipid and Glucose Study (TLGS) [11,12] is a community-based prospective study preformed in residents of district no.13 of Tehran, the capital city of Iran. This study, which began in 1999, originally designed to detect and prevent non-communicable diseases and their risk factors. In this study, the baseline measurements are being followed every three years. Of 1454 individuals aged 10 to 19 years completed the examination in the fourth TLGS Follow-up Survey (2008-2011), 848 were randomly selected for dietary assessment. After excluding participants with incomplete data on anthropometric, biochemical or physical activity, had special diets, used thyroid or lipid regulators medications, and over- and underreporters, the final sample-size was 717 (391 girls and 326 boys). Informed written consent was obtained from the parents of those aged $<18$ of age and from all those aged $\geq$ 18 years. The study protocol was approved by the ethics committee of the Research Institute for Endocrine Science, Shahid Beheshti University of Medical Sciences.

\subsection{Dietary Assessment}

Dietary data were collected by means of a validated 168-item semi-quantitative Food Frequency Questionnaire (FFQ) $[13,14,15]$. Trained dietitians asked participants to designate their consumption frequency for each food item consumed during the previous year on a daily, weekly, or monthly basis. Portion sizes of consumed foods that were specified according to the household measures were converted to grams [16]. Then, daily intake for each FFQ food item was calculated in gram. Because the Iranian Food Composition Table (FCT) [17] provides a few data to analyze foods and beverages for energy and nutrients, we used the FCT of the US Department of Agriculture (USDA) [18]. However, for some Iranian food items that are not listed in the USDA FCT (e.g. kashk) the Iranian FCT was used. To calculate trans fatty acid content of foods (not included in USDA FCT), Dorosti food composition table book [19] was used. In addition, the nutrient content of mixed food items was calculated based on usual restaurant recipes.

\subsection{Biological Measurement or Other Measures}

For measurement of lipid concentrations of each participant, a fasting blood sample was drawn between 7:00 and 9:00 Am, after 12-14 hours of overnight fasting. Samples were centrifuged within 30 to 45 minutes of collection.

All blood analyses were done at the TLGS research laboratory, on the same day of blood collection. Blood samples were analyzed using selectra 2 auto-analyzer (Vital Scientific, Spankeren, Netherlands) and commercial kits (Pars Azmoon Inc., Tehran, Iran). Triglyceride (TG) concentration was assessed using an enzymatic calorimetric method, with glycerol phosphate oxidase. Inter- and intra-assay coefficients of variation were $1.6 \%$ and $0.6 \%$, respectively. TC was measured with cholesterol esterase and cholesterol oxidase by using enzymatic colorimetric method. After precipitation of the apolipoprotein $\beta$ containing lipoproteins with posphotungstic acid, the HDL-C was assessed. Inter- and intra-assay CV for both TC and HDL-C were $0.5 \%$ and $2 \%$ respectively. LDL-C was calculated from the serum TC, TG and HDL-C concentrations expressed in mg/dL, using the Friedewald formula [20]. Monitoring of assay performance was performed once every 20 tests using lipid control serum, Percinorm (reference range), and Percipath (pathologic range) wherever applicable (Boehringer Mannheim, Mannheim, Germany; catalog no. 1446070 for Percinorm and 171778 for Percipath) [11].

\subsection{Statistical Analysis}

All statistical analysis was performed using the SPSS software (version 20.0). P value $<0.05$ was considered statistically significant. To compare the characteristics of participants between two sex t-test was used. Analysis of covariance preformed to assess mean values of serum lipids level across the percent of energy intake from fat and fatty acids quartiles, adjusted for sex and age. To determine $\mathrm{P}_{\text {trend }}$ within quartile categories, linear regression coefficient was used.

\section{Results}

Our 717 participants included 326 boys and 391 girls with a mean age of 14.7 years. Characteristics of these adolescents were shown in Table 1. Serum TG concentration was higher in boys, but there were no significant difference in serum concentration of TC, LDL$\mathrm{C}$ and HDL-C between two sexes.

Table 1. Characteristics of Tehranian adolescents in specified sex groups ${ }^{\text {a }}$

\begin{tabular}{|c|c|c|c|c|}
\hline & $\begin{array}{c}\text { total } \\
(n=717)\end{array}$ & $\begin{array}{c}\text { Girls } \\
(n=391)\end{array}$ & $\begin{array}{c}\text { Boys } \\
(n=326)\end{array}$ & $\mathrm{P}$ value \\
\hline Age (year) & $14.7 \pm 2.9$ & $14.9 \pm 2.8$ & $14.5 \pm 2.9$ & 0.06 \\
\hline Weight (kg) & $56.0 \pm 16.5$ & $53.8 \pm 13.4$ & $58.6 \pm 19.3$ & $<0.001$ \\
\hline Height (cm) & $158.6 \pm 12.1$ & $155.6 \pm 8.7$ & $162.1 \pm 14.4$ & $<0.001$ \\
\hline $\mathrm{TG}^{\mathrm{b}}(\mathrm{mg} / \mathrm{dl})$ & $96.3 \pm 48.7$ & $92.1 \pm 45.6$ & $101.2 \pm 51.9$ & 0.01 \\
\hline $\mathrm{TC}^{\mathrm{c}}(\mathrm{mg} / \mathrm{dl})$ & $156.1 \pm 28.6$ & $155.7 \pm 25.4$ & $156.6 \pm 30.0$ & 0.66 \\
\hline $\mathrm{HDL}_{-\mathrm{C}}{ }^{\mathrm{e}}(\mathrm{mg} / \mathrm{dl})$ & $49.9 \pm 10.7$ & $50.6 \pm 10.6$ & $49.1 \pm 10.8$ & 0.73 \\
\hline
\end{tabular}

\footnotetext{
${ }^{\mathrm{a}}$ Values are determined using T-test

b Triglycerides

c Total Cholesterol

${ }^{\mathrm{d}}$ Low Density Lipoprotein Cholesterol

${ }^{\mathrm{e}}$ High Density Lipoprotein Cholesterol
} 
In Table 2, percents of energy intake from total fat and various types of fatty acids were shown. In girls, the percent of energy intake from total fat, PUFA, MUFA and trans fatty acids were higher significantly.

Table 2. Mean \pm SD dietary intake of participants in specified sex groups ${ }^{\text {a }}$

\begin{tabular}{|c|c|c|c|c|}
\hline & $\begin{array}{c}\text { total } \\
(n=716)\end{array}$ & $\begin{array}{c}\text { Girls } \\
(n=391)\end{array}$ & $\begin{array}{c}\text { Boys } \\
(n=325)\end{array}$ & $P$ value \\
\hline Total reported energy(kcal/d) & $2726 \pm 833$ & $2636 \pm 804$ & $2833 \pm 855$ & 0.002 \\
\hline Fat (\% of energy intake) & $30.6 \pm 6.0$ & $31.2 \pm 6.1$ & $30.0 \pm 5.8$ & 0.01 \\
\hline $\mathrm{SFA}^{\mathrm{b}}$ (\% of energy intake) & $10.3 \pm 2.9$ & $10.2 \pm 2.9$ & $10.4 \pm 2.9$ & 0.57 \\
\hline PUFA $^{\mathrm{c}}$ (\% of energy intake) & $6.1 \pm 1.8$ & $6.3 \pm 1.9$ & $5.8 \pm 1.7$ & 0.001 \\
\hline MUFA $^{\mathrm{d}}$ (\% of energy intake) & $10.1 \pm 2.6$ & $10.3 \pm 2.8$ & $9.8 \pm 2.2$ & 0.005 \\
\hline
\end{tabular}

${ }^{\mathrm{a}}$ Values are determined using t-test

b Saturated fatty acid

${ }^{\mathrm{c}}$ Poly unsaturated fatty acid

d Mono unsaturated fatty acid.

Adjusted mean values of serum lipid levels across the quartile categories of percent of calorie from total fat and types of fatty acids were given in Table 3. After adjustment for sex and age serum TG level showed a significant decreasing trend according to the quartiles of percent of energy intake from PUFA. Moreover, serum
HDL-C level increased significantly according to the quartiles of percent of energy intake from PUFA. No significant difference were observed between quartiles categories of percent of calorie from total fat and other types of fatty acids with serum lipid profile.

Table 3. Mean (SE) for serum lipid levels in participants across the quartile categories of percent of calorie form dietary fat components a

\begin{tabular}{|c|c|c|c|c|}
\hline & TG (mg/dl) & TC (mg/dl) & LDL-C (mg/dl) & HDL-C (mg/dl) \\
\hline \multicolumn{5}{|c|}{ Total reported energy(kcal/d) } \\
\hline Q1 & $92.9(3.6)$ & $154.3(2.1)$ & $84.4(1.8)$ & $50.6(0.8)$ \\
\hline Q2 & $96.8(3.6)$ & $157.4(2.1)$ & $87.4(1.8)$ & $50.6(0.8)$ \\
\hline Q3 & $100.5(3.6)$ & $157.5(2.1)$ & $87.9(1.8)$ & $49.8(0.8)$ \\
\hline Q4 & $94.6(3.6)$ & $155.0(2.1)$ & $87.2(1.8)$ & $48.6(0.8)$ \\
\hline \multicolumn{5}{|c|}{ Total Fat (\% of energy intake) } \\
\hline Q1 & $98.2(3.8)$ & $156.3(2.2)$ & $86.0(1.9)$ & $50.1(0.8)$ \\
\hline Q2 & $94.4(3.8)$ & $157.1(2.2)$ & $87.4(1.9)$ & $50.8(0.8)$ \\
\hline Q3 & $97.4(3.5)$ & $154.3(2.0)$ & $85.4(1.8)$ & $48.6(0.7)$ \\
\hline Q4 & $96.2(3.4)$ & $156.7(2.0)$ & $88.4(1.8)$ & $50.2(0.7)$ \\
\hline \multicolumn{5}{|c|}{ SFA $^{\text {b }}$ (\% of energy intake) } \\
\hline Q1 & 98.5 (3.6) & $158.1(2.1)$ & $87.1(1.8)$ & $50.9(0.8)$ \\
\hline Q2 & 91.4 (3.9) & $155.4(2.3)$ & $85.8(2.0)$ & $50.7(0.8)$ \\
\hline Q3 & $97.9(3.3)$ & $155.7(1.9)$ & $87.9(1.7)$ & $49.2(0.7)$ \\
\hline Q4 & $96.1(3.6)$ & $155.0(2.1)$ & $86.2(1.8)$ & $48.9(0.7)$ \\
\hline \multicolumn{5}{|c|}{ PUFA $^{\mathrm{c}}$ (\% of energy intake) } \\
\hline Q1 & $101.1(3.5)$ & $154.3(2.1)$ & $84.0(1.8)$ & $49.3(0.8)$ \\
\hline Q2 & $99.8(3.6)$ & $156.9(2.1)$ & $88.6(1.8)$ & $49.4(0.8)$ \\
\hline Q3 & $100.4(3.6)$ & $157.5(2.1)$ & $88.0(1.8)$ & $49.0(0.8)$ \\
\hline Q4 & $84.0(3.6)^{e}$ & $155.6(2.1)$ & $86.9(1.8)$ & $51.7(0.8)^{\mathrm{e}}$ \\
\hline \multicolumn{5}{|c|}{ MUFA $^{d}$ (\% of energy intake) } \\
\hline Q1 & $98.9(3.6)$ & $157.1(2.1)$ & $86.9(1.8)$ & $50.3(0.8)$ \\
\hline Q2 & 96.5 (3.6) & $153.9(2.1)$ & $85.2(1.8)$ & $50.2(0.8)$ \\
\hline Q3 & $100.4(3.6)$ & $156.2(2.1)$ & $86.0(1.8)$ & $49.4(0.8)$ \\
\hline Q4 & $88.7(3.6)$ & $156.9(2.1)$ & $89.1(1.8)$ & $49.8(0.8)$ \\
\hline \multicolumn{5}{|c|}{ Trans fatty acids (\% of energy intake) } \\
\hline Q1 & 99.5 (3.6) & $156.4(2.1)$ & $86.4(1.8)$ & $49.9(0.8)$ \\
\hline Q2 & $97.8(3.6)$ & $157.0(2.1)$ & $86.8(1.8)$ & $50.7(0.8)$ \\
\hline Q3 & $97.0(3.6)$ & $154.8(2.1)$ & $87.1(1.8)$ & $48.5(0.8)$ \\
\hline Q4 & $90.8(3.6)$ & $156.1(2.1)$ & $87.1(1.8)$ & $50.4(0.8)$ \\
\hline
\end{tabular}

\footnotetext{
${ }^{\mathrm{a}}$ Values are determined using ANCOVA after adjustment for age and sex

b Saturated fatty acid

${ }^{c}$ Poly unsaturated fatty acid

${ }^{\mathrm{d}}$ Mono unsaturated fatty acid

${ }^{\mathrm{e}} \mathrm{P}$ for trend $<0.05$ (determined using linear regression).
} 


\section{Discussion}

The existing evidence strongly supports beginning primary prevention of atherosclerotic disease in childhood [21]. The dietary fat intake of adolescents plays an important role in their growth and development, and also has a long-term effect on adult health. For this reason, both quality and quantity of dietary fat must be considered [9]. In recent years, there has been increasing concern about dietary effects on dyslipidemia, because it can be a major contributor to increase risk of CVD [22]. In the past, reducing saturated fatty acid intake, as a means of lowering LDL-C concentrations, was the main focus of dietary recommendations for CVD prevention and treatment [7]. Siri and colleagues [8] have shown that saturated fat only affect serum cholesterol when less than 5 percent of energy intake provided from PUFA. Also, they have indicated that replacement of saturated fat by PUFA or MUFA lowers both LDL-C and HDL-C. Another studies showed that among different types of fatty acids, trans and saturated fatty acids have the worst effect on blood lipids, increased LDL and decreased HDL cholesterol $[9,23]$. In addition, it has been shown that higher dose of omega 3 fatty acids significantly lowered serum TG concentration [2].

In the present study, among different types of fatty acid intakes, only those in the highest quartile category of PUFA showed a significant decreasing trend in serum TG and increasing trend in serum HDL-C. Other type of fatty acids did not show a significant association with lipid profiles. Like others developing countries, Iran is experiencing a rapid nutritional transition including increased consumption of western foods instead of traditional foods intake, such as plant sterols and dietary fiber that are clearly having a role in lipid metabolism [24]. Previous investigations have shown that dyslipidemia in Iranian adolescents can be attributed mainly to improper dietary habits, especially in respect of fast foods and snacks that contain high levels of saturated and trans fatty acids, but are usually preferred to the traditional family meals and/or snacks [9].

As shown in the past studies, prevalence of overweight and obesity is increasing in Iranian adolescents (prevalence of large waist circumference has increased from $12.2 \%$ in $1999-2001$ to $51.8 \%$ in $2008-2011$ ) [25,26]. This indicated that during nutrition transition incorrect eating habits have lead to increased energy intake more than the amount of required and thus overweight and obesity. Obesity is associated with changes in the serum lipid profiles [27]. Therefore, in this population overconsumption can be considered as a cause of not observed correlation between intake of saturated and trans fatty acids and levels of blood lipids. In other words, intake of energy more than the amount of required and thus overweight and obesity, fades impacts of types of fatty acids on serum lipid profile.

These findings are subject to some potential limitations. Because of the cross-sectional design of the present study, it is impossible to know causal relations and whether some participants altered their diet. In addition, since Iranian Food Composition Table (FCT) is incomplete, we used the FCT of the US Department of Agriculture (USDA) to analyze foods and beverages for energy and nutrients. An important limitation to consider in interpretation of our results is the use of FFQ for collecting the dietary data, although the FFQ used has previously been shown to be valid $[13,14]$. However, the strength of the present study is being a population-based analysis and conducted in a developing country under nutrition transition.

In conclusion, because of the importance of dietary practices, it is necessary that healthy guidelines be developed specifically for each country. To our knowledge, this is the first study to assess the relationship between intake of dietary fat and serum lipid profiles in Iranian adolescents. Although the percent of calorie intake from fat and different types of fatty acids have association with serum lipids levels, but the amount of total energy intake plays more important role in lipid profiles.

\section{Acknowledgment}

We are grateful to the TLGS participants for their collaboration. This study was supported by a grant from the Research Institute of Endocrine Sciences, Shahid Beheshti University Medical Sciences, Tehran, Iran.

\section{References}

[1] Haney EM, Huffman LH, Bougatsos C, Freeman M, Steiner RD, Nelson HD, "Screening and treatment for lipid disorders in children and adolescents: systematic evidence review for the US Preventive Services Task Force", Pediatrics, 120(1), 189-214, 2007.

[2] Skulas-Ray AC, Kris-Etherton PM, Harris WS, Heuvel JPV, Wagner PR, West SG, "Dose-response effects of omega-3 fatty acids on triglycerides, inflammation, and endothelial function in healthy persons with moderate hypertriglyceridemia" The American journal of clinical nutrition, 93(2), 243-252, 2011.

[3] Strong JP, Malcom GT, McMahan CA, Tracy RE, Newman III WP, Herderick EE, et al, "Prevalence and extent of atherosclerosis in adolescents and young adults: implications for prevention from the Pathobiological Determinants of Atherosclerosis in Youth Study", Jama, 281(8), 727-735, 1999.

[4] Valente AM, Newburger JW, Lauer RM, "Hyperlipidemia in children and adolescents", American heart journal, 142(3), 433439, 2001.

[5] Haas G-M, Liepold E, Schwandt P, "Metabolic risk factors, leisure time physical activity, and nutrition in german children and adolescents", Cholesterol, 2012, 1-3, 2012.

[6] Dias C, Garg R, Wood L, Garg M, "Saturated fat consumption may not be the main cause of increased blood lipid levels", Medical hypotheses, 82(2), 187-195, 2013.

[7] Astrup A, Dyerberg J, Elwood P, Hermansen K, Hu FB, Jakobsen $\mathrm{MU}$, et al, "The role of reducing intakes of saturated fat in the prevention of cardiovascular disease: where does the evidence stand in 2010?", The American journal of clinical nutrition, 93(4), 684-688, 2011.

[8] Siri-Tarino PW, Sun Q, Hu FB, Krauss RM, "Saturated fat, carbohydrate, and cardiovascular disease", The American journal of clinical nutrition, 91(3), 502-509, 2010.

[9] Kelishadi R, Pour MH, Zadegan NS, Kahbazi M, Sadry G, Amani A, et al, "Dietary fat intake and lipid profiles of Iranian adolescents: Isfahan Healthy Heart Program-heart health promotion from childhood", Preventive medicine, 39(4), 760-766, 2004.

[10] Kelishadi R, Hashemipour M, Sarraf-Zadegan N, Amiri M, "Trend of atherosclerosis risk factors in children of Isfahan", Asian cardiovascular and thoracic annals, 9(1), 36-40, 2001.

[11] Azizi F, Ghanbarian A, Momenan AA, Hadaegh F, Mirmiran P, Hedayati M, et al, "Prevention of non-communicable disease in a population in nutrition transition: Tehran Lipid and Glucose Study phase II", Trials, 10(5), 1-15, 2009.

[12] Azizi F, Rahmani M, Emami H, Mirmiran P, Hajipour R, Madjid $\mathrm{M}$, et al, "Cardiovascular risk factors in an Iranian urban 
population: Tehran lipid and glucose study (phase 1)", Social and Preventive Medicine, 47(6), 408-426, 2002.

[13] Hosseini Esfahani F, Asghari G, Mirmiran P, Azizi F, "Reproducibility and relative validity of food group intake in a food frequency questionnaire developed for the Tehran lipid and glucose study", Journal of Epidemiology, 20(2), 150-158, 2010.

[14] Mirmiran P, Hosseini Esfahani F, Mehrabi Y, Hedayati M, Azizi F, "Reliability and relative validity of an FFQ for nutrients in the Tehran Lipid and Glucose Study", Public health nutrition, 13(5), 654-662, 2010.

[15] Asghari G, Rezazadeh A, Hosseini-Esfahani F, Mehrabi Y, Mirmiran P, Azizi F, "Reliability, comparative validity and stability of dietary patterns derived from an FFQ in the Tehran Lipid and Glucose Study", British Journal of Nutrition, 108(06), 1109-1117, 2012.

[16] Ghaffarpour M H-RA, Kianfar H, "The manual for household measures, cooking yields factors and edible portion of food, Tehran, Keshaverzi press, 1999.

[17] Azar M, Sarkisian E, Food Composition Table of Iran, National Nutrition and Food Research Institute. Shahid Beheshti University Press, Tehran, 1980.

[18] The Nutrient Data Laboratory. Food Composition Table (FCT), food and nutrition information center, United States Department of Agriculture (USDA). Available from: URL: http://www.nal.usda.gov/fnic/foodcomp.

[19] Dorosti A, Tabatabaii M, food composition table, tehran, donyaye taghzieh [in Persian], 2007.

[20] Friedewald WT, Levy RI, Fredrickson DS, "Estimation of the concentration of low-density lipoprotein cholesterol in plasma, without use of the preparative ultracentrifuge", Clinical chemistry,18(6), 499-502, 1972.

[21] Kavey R-EW, Daniels SR, Lauer RM, Atkins DL, Hayman LL, Taubert K, "American Heart Association guidelines for primary prevention of atherosclerotic cardiovascular disease beginning in childhood", Circulation,107(11), 1562-1566, 2003.

[22] Krauss RM, Siri PW, "Metabolic abnormalities: triglyceride and low-density lipoprotein. Endocrinology and metabolism clinics of North America", 33(2), 405-415, 2004.

[23] Brouwer IA, Wanders AJ, Katan MB, "Effect of animal and industrial trans fatty acids on HDL and LDL cholesterol levels in humans-a quantitative review", PLoS One, 5(3), e9434, 2010.

[24] Ghassemi H, Harrison G, Mohammad K, "An accelerated nutrition transition in Iran", Public health nutrition, 5(1a), 149-155, 2002.

[25] Mirmiran P, Sherafat-Kazemzadeh R, Farahani SJ, Asghari G, Niroomand M, Momenan AA, et al, "Performance of different definitions of metabolic syndrome for children and adolescents in a 6-year follow-up: Tehran Lipid and Glucose Study (TLGS)", Diabetes research and clinical practice, 89(3), 327-333, 2010.

[26] Sahar Mohseni-Takalloo PM, Firoozeh Hosseini-Esfahani, Yadollah Mehrabi, and Fereidoun Azizi, "Metabolic Syndrome and its Association with Healthy Eating Index-2005 in Adolescents: Tehran Lipid and Glucose Study", Journal of Food and Nutrition Research,2(4), 155-161, 2014.

[27] Pietiläinen KH, Sysi-Aho M, Rissanen A, Seppänen-Laakso T, Yki-Järvinen H, Kaprio J, et al, "Acquired obesity is associated with changes in the serum lipidomic profile independent of genetic effects-a monozygotic twin study", PloS one,2(2), e218, 2007. 